\title{
Robotics: the future of liver surgery?
}

Minimally invasive surgery, sometimes known as keyhole surgery, has proven benefits over traditional open surgery. One type of minimally invasive surgery involves the use of high-tech robotics. Dr Alejandro Mella of the Methodist DAgestive Institure, Dallas, TX, USA, is invasive liver surgery. Along with his team, Dr Mejia uses his first-hand knowledge to investigate different surgical techniques in order to provid the best possible outcomes for his patients. His work takes an in-depth look at the various liver surgery DrMe Mija found that robotic surgery has som important clinical advantages.

he traditional image of a scalpel-
wielding surgeon is rapidly wielding surgeon is rapidly the long-established methods of open surgery, many physicians now prefer to use minimally invasive techniques where possible.

One common minimally invasive method is laparoscopic surgery, which This technique uses an instrument called a laparoscope. A laparoscope is a long thin tube with a light and a camera. The laparoscope is inserted through a small incision and the camera captures video that the surgeon views on a screen. This allows the surgeon to carry out the operation without making a large surgica incision, as would be typical in open surgery. For the patient, laparoscopic surgery normally means a shorter recov time and less pain, bleeding and scarring compared to traditional open surge

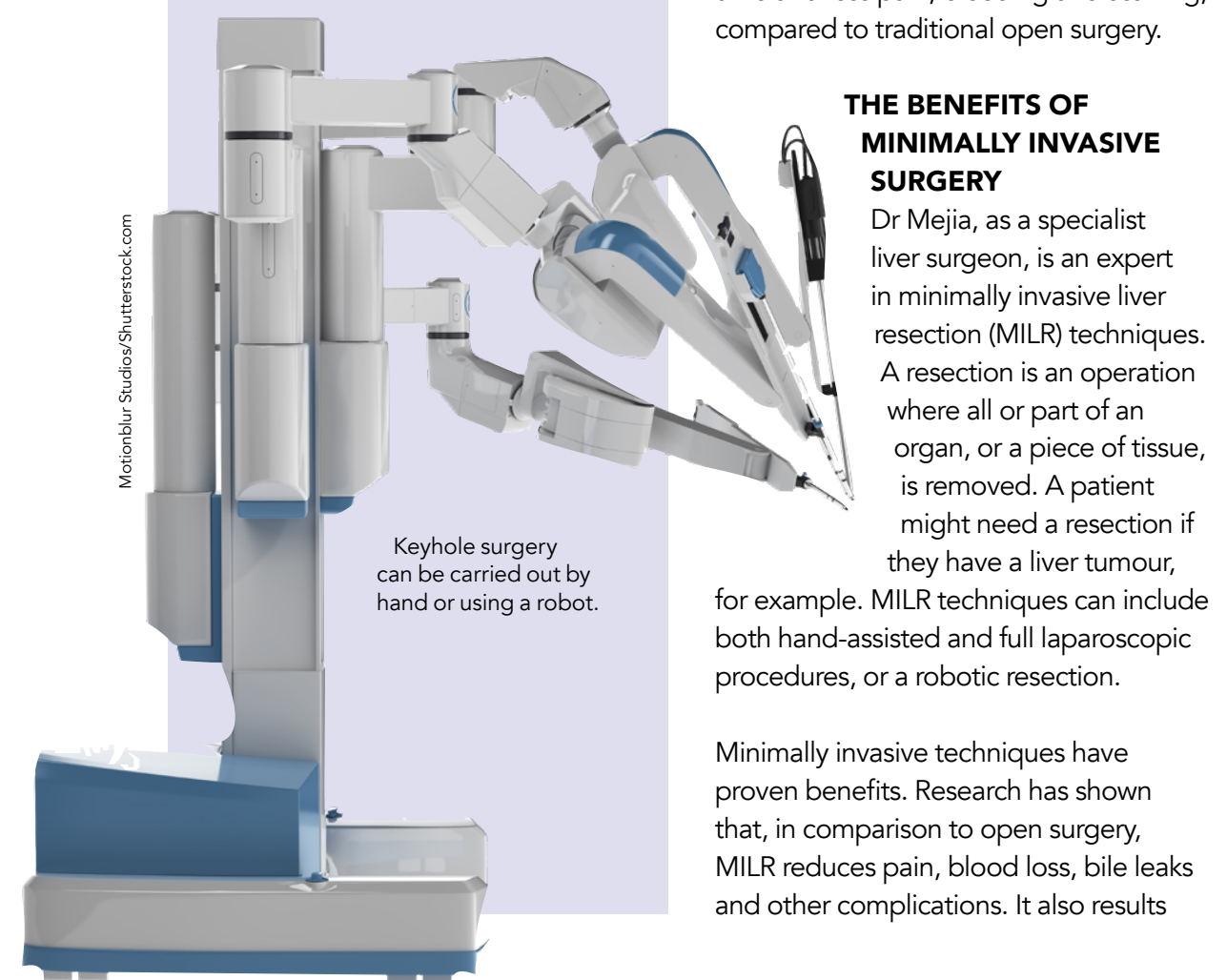

in a shorter hospital stay. Although some studies have suggested that the robotic approach gives the best outcomes for the patient, exactly which type of MILR - laparoscopic or robotic - produces

To try to answer this question, Dr Mejia and his colleagues decided to compare the different types of MILR. They aimed to of hospital resources for three different MILR techniques.

ADVANCING LIVER SURGERY: WHICH MINIMALLY INVASIVE TECHNIOUE IS BEST? Dr Mejia and his team compared full laparoscopic, hand-assisted and robotic liver resections. Full laparoscopic surgery relies solely on the laparoscope and other instruments inserted through one or more small incisions. In contrast, hand-assisted laparoscopic surgery allows the surgeon to use their non-dominant hand, via a procedure. This car improve the of the procedure. This can improve the surgeon's providing tactile feedback. Finally,

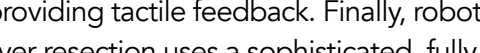
robotic surgical system. The camera and other surgical instruments are operated by the robot, which the surgeon controls from a console.

Dr Mejia and his colleagues examined a total of 214 liver resections that took place at the Methodist Dallas Medical Centre between 2005 and 2018. This included 98 fully laparoscopic cases, 73 handassisted and 43 robotic resections. The and 2018

Two types of resection were considered. Minor resections are those in which

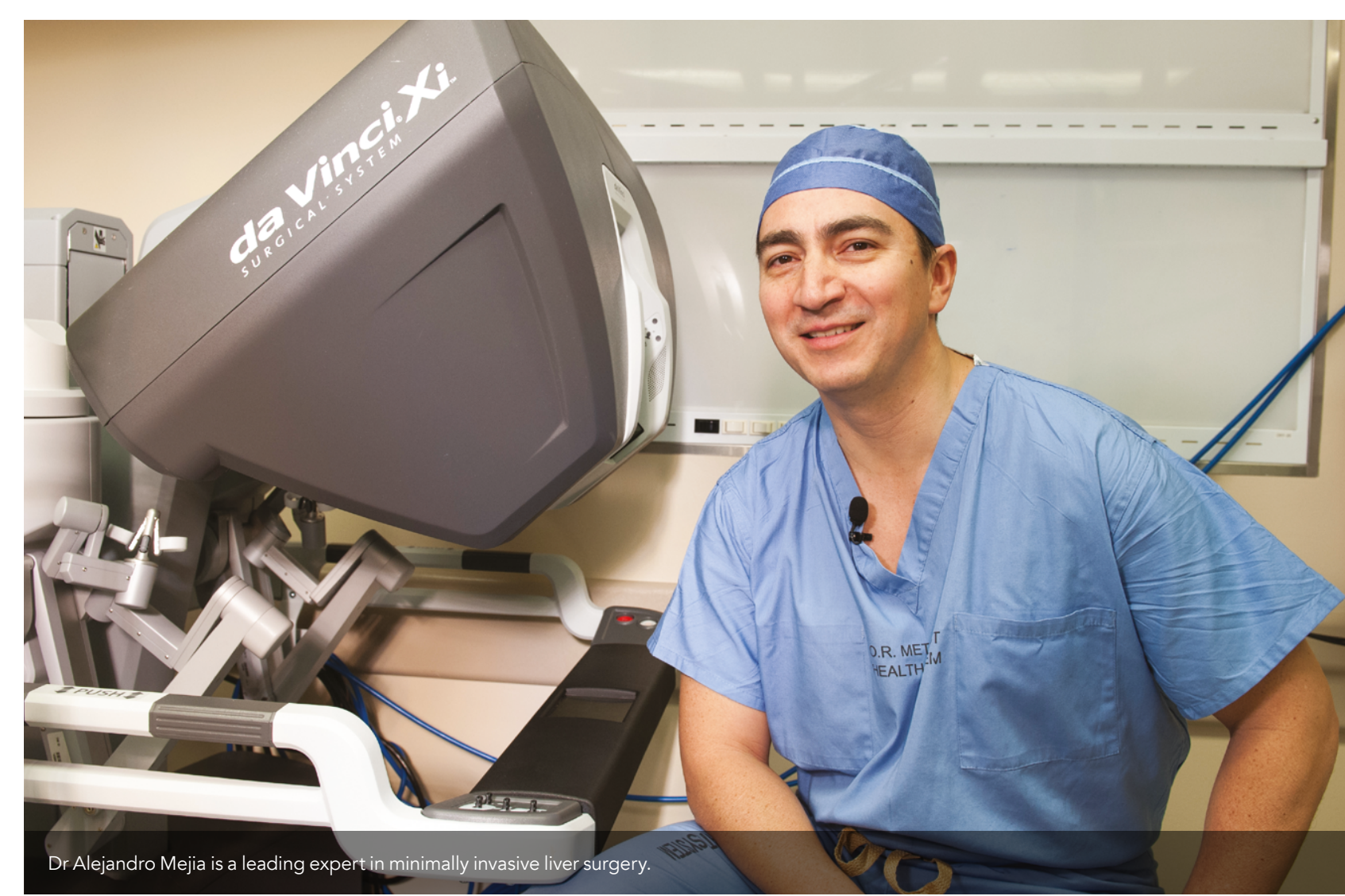

Dr Mejia believes that, for minor resections, the robotic technique will become the standard of care.

segments of the liver) are removed. Major The team also found that hand-assisted resection involves the removal of three or more segments. The patients in the study
included 160 cases of minor resection and 54 cases of major resection.

As the team is based in the US, they also looked at room and board charges to compare the demands on hosp resources arising from each of the three types of procedure.

\section{ROBOTIC SURGERY}

HAS CLEAR BENEFITS

Dr Mejia and his team found that patients who underwent robotic surgery for min resections had a significantly shorter hospital stay, compared to hand-assist laparoscopic surgery patients. This supports the results of previous studies, which have found shorter lengths of stay for robotic surgery cases. However, Drifferences bedy is the first to compare differences between $m$.
resections using MILR.
The the robotic surgery, meaning that patients spent longer in the operating theatre time spent in the operating room was 181 minutes, around 50 minutes longer than for robotic surgery.

Overall, hospital charges were lowest for patients who were treated with full laparoscopic surgery for a minor resection. As only 54 cases of major resection were investigated, the researchers were able to draw fewer conclusions. However, they did discover that, for major resection patients, the full laparoscopic group experienced the least blood loss, and also incurred the lowest charges for room and board.

WHAT DRIVES THE BEST CLINICAL OUTCOMES? The researchers suggest that outcomes for the patients may depend more on the type of resection - major or minor - than the MILR method chosen by the surgeon. Nevertheless, Dr Mejia believes that, for minor resections, the robotic technique gives the best results. A key factor in this opin th is the reduced length of hospital sta tor these patients, a good indicator of a shorter recovery time. As well as the the robots - including magnifeation, dextery and $3 \mathrm{D}$ optics - give a clear advantage over the other types of MILR.

Robotic surgery may well also give the best results for major resection patients. However, more research is needed for a definitive

\section{BALANCING THE} COSTS VS BENEFITS

Ferating costs were higher for robotic surgery (even without including

the expensive annual maintenance

fees for the equipment) than for full laparoscopic surgery. There was little difference in charges between robotic and hand-assisted laparoscopic surgery rowever, patients who underwent robotic surgery incurred lower pharma 


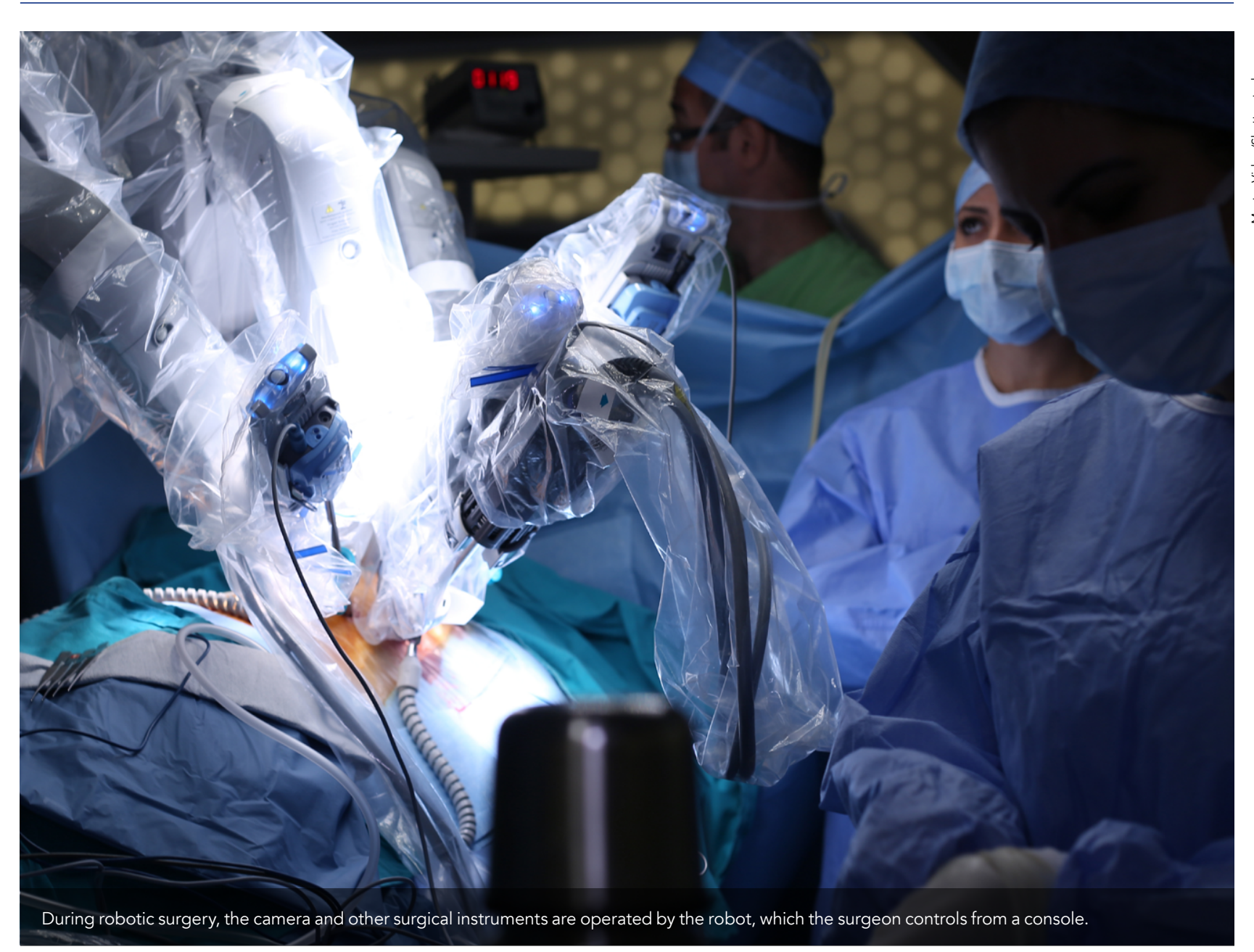

pain, and needed less pain relief, than other patients.

In another benefit for robotic surgery, major resection patients had less need for respiratory therapy. This suggests robotic surgery patients suftered less
respiratory discomfort and/or a quicker respiratory discont

\section{THE LIVER SURGERY}

LEARNING CURVE

The researchers acknowledge that their study has a few limitations. The sample sizes of both the robotic surgery and major resection groups were relatively small. Also, the surgeons who carried out the operations would have experienced learning curve over the 13 years covered by the study. As all the patients were operated on by the same surgical tear those in the later years of the study would have benefitted from many years of accumulated experience.

While the robotic technique seems to produce the best results for mino
resections, in this study all MLLR

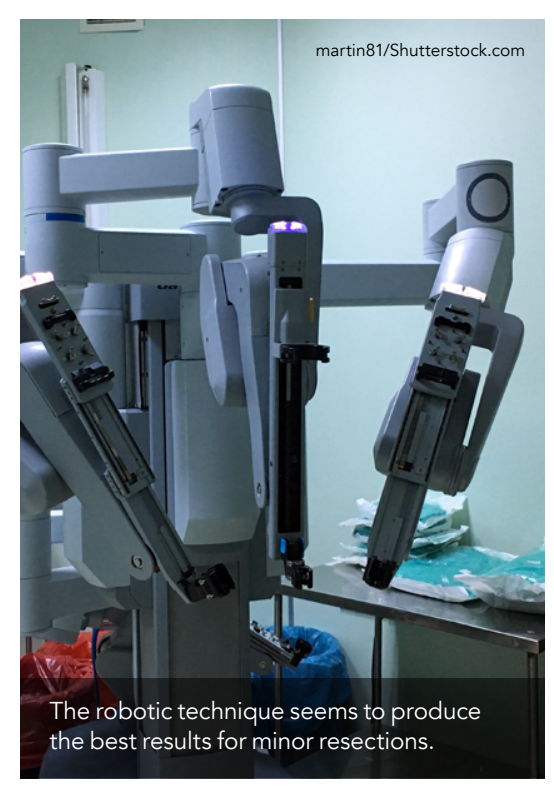

Patients who underwent robotic surgery for minor resections went home faster. methods gave comparable results for major resections. However, as already mentioned, the sample size for major resections was small. Also, most major resections included in this study aparoscopic techniques.

Dr Mejia and his team emphasise that needed than on which type of MIR is chosen by the surgeon. Even though robotic surgery has some clear clinica benefits, all types of MILR actually resulted in very good outcomes. No one technique appears to be greatly superior to the others.

So, what is the next step? Dr Mejia needed in order to decide which of these meantime, the benefits of robotic surgery fewer complications, a faster recovery in the operating theatre. patient outcomes may depend more suggests that randomised clinical trials are three techniques is truly the best. In the and a shorter hospital stay - demonstrate

\section{Behind the Research} Dr Alejandro Mejia

E: AlejandroMejia@mhd.comＥ: ElainaVivian@mhd.comＴ: +1 6364260708 or +12149336611

W: https://www.methodisthealthsystem.org/methodist-dallas-medical-center/medical-services/gastroenterology/methodist-digestive institute/\# $x 1 y 138$

W: https://www.methodisthealthsystem.org/methodist-dallas-medical-center/medical-services/the-liver-institute/\# $\sim$ 1y1381

W: https://www.methodisthealthsystem.org/methodist-dallas-medical-center/medical-services/cancer-services/treatments/pancreaticcancer-treatment/robotic-whipple-for-pancreatic-cancer/

W: https://www.methodisthealthsystem.org/methodist-dallas-medical-center/medical-services/pancreatic-cancer/pancreatic-care-team/

\section{Research Objectives}

Dr Mejia's work compares approaches to minimally invasive liver resection (MILR) to examine whether a robotic

\section{Detail}

Elaina Vivian, Methodist Digestive Institute 441 N Beckley Avenue, Dallas, TX 75203, USA

Bio

Dr Alejandro Mejia received his medical training at the Universidad Pontificia Bolivariana, Colombia. He was trained as a general surgeon at the University of Texas Health Science Center at San Antonio and then completed a fellowship in multi-organ abdominal transplantation and hepatobiliary surgery at the University of Nebraska Medical Centerin Omaha. There he trained extensively in splitliver and live-donor liver transplantation. He served as an assistant professor at the University of Texas at San Antonio prior to joining Methodist Dallas Medical Center. He is certified by the American Board of Surgery and is a member of the American Society of Transplant Surgeons. He led a team that performed the first fully robotic Whipple and major liver resection in North Texas. He is currently President of The Texas Transplantation Society. His main interests include adult liver transplantation, hepatobiliary, and pancreatic surgery. Methodist Dallas is one of only a handful of centres in the country recognised by Intuitive Surgical as a case observation Epicenter for minimally invasive hepatobiliary surgery.

Collaborators

Stephen Cheng, Mrs Elaina Vivian, Mr Jimmy Shah, Mrs Hellen Oduor, and Mrs Priyanka Acharya.

\section{References}

Mejia, A. et al. 2019. Minimally invasive liver resection in the ra of robotics: analysis of 214 cases. Surgical Endoscopy doi: 10.1007/s00464-019-06773-3.

\section{Personal Response}

Are there any new or expected developments in the field of robotic surgery that might change how liver surgery is performed in the coming years?

II All signs point to robotics becoming the standard in to show a clear benefit As a high-volume centre, we will be able to contribute considerable data to these efforts.

In the future, improved visualisation of the liver using 3D robotically navigate with greater precision improving the surgeon's ability to obtain sufficient margins while at the same time sparing healthy tissue.

\section{II}

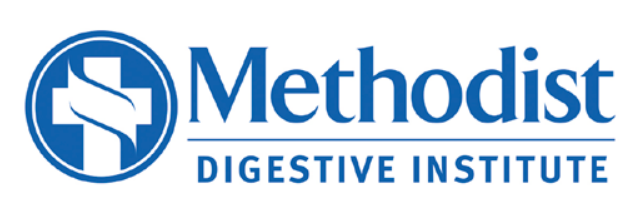

